\title{
Cuidados de enfermagem à luz da complexidade: uma revisão integrativa
}

\author{
Nursing care in the light of complexity: an integrative review \\ Atención de enfermería a la luz de la complejidad: una revisión integrativa
}

Recebido: 21/01/2021 | Revisado: 24/01/2021 | Aceito: 26/01/2021 | Publicado: 04/02/2021

\author{
Patrícia Bossolani Charlo \\ ORCID: https://orcid.org/0000-0002-8262-2086 \\ Universidade Estadual de Maringá, Brasil \\ E-mail: patbcs20@gmail.com \\ Luana Cristina Bellini Cardoso \\ ORCID: https://orcid.org/0000-0001-8460-1177 \\ Universidade Estadual de Maringá, Brasil \\ E-mail: luana.bellini@hotmail.com \\ Graziele Adrieli Rodrigues Pires \\ ORCID: https://orcid.org/0000-0002-9673-9218 \\ Universidade Estadual de Maringá, Brasil \\ E-mail: graziele_rodriguespires@hotmail.com \\ Cremilde Aparecida Trindade Radovanovic \\ ORCID: https://orcid.org/0000-0001-9825-3062 \\ Universidade Estadual de Maringá, Brasil \\ E-mail: kikanovic2010@ hotmail.com \\ Lígia Carreira \\ ORCID: https://orcid.org/0000-0003-3891-4222 \\ Universidade Estadual de Maringá, Brasil \\ E-mail: ligiacarreira.uem@gmail.com \\ Betina Horner Schlindwein Meirelles \\ ORCID: https://orcid.org/0000-0003-1940-1608 \\ Universidade Federal de Santa Catarina, Brasil \\ E-mail: betina.hsm@ufsc.br \\ Maria Aparecida Salci \\ ORCID: https://orcid.org/0000-0002-6386-1962 \\ Universidade Estadual de Maringá, Brasil \\ E-mail: masalci@uem.br
}

\begin{abstract}
Resumo
O objetivo deste estudo foi identificar a utilização do referencial teórico da Complexidade proposto por Edgar Morin na produção do conhecimento sobre cuidado de enfermagem. Trata-se de uma revisão integrativa da literatura, realizada nas bases de dados Biblioteca Virtual em Saúde e PubMed, no período de 2009 a 2020. Para a seleção dos artigos utilizou- a estratégia PICo. Foram incluídos artigos completos e originais. Para auxiliar a análise dos dados, as informações foram operacionalizadas no software IRAMUTEQ®. Foram selecionados 14 artigos, publicados em periódicos nacionais e internacionais, com predomínio de pesquisas qualitativas. Após análise minuciosa originou-se duas classes - Classe 1: O paradigma da Complexidade e os cuidados de enfermagem diante as adversidades da assistência. Classe 2: Complexidade dos cuidados nas fases da infância e adolescência. A Teoria da Complexidade é utilizada para compreender as relações e os fenômenos que circundam a assistência de enfermagem como um todo.
\end{abstract}

Palavras-chave: Enfermagem; Saúde; Cuidados de enfermagem; Assistência integral à saúde.

\begin{abstract}
The aim of this study was to identify the use of the theoretical framework of Complexity proposed by Edgar Morin in the production of knowledge about nursing care. This is an integrative literature review, carried out in the Virtual Health Library and PubMed databases, from 2009 to 2020. For the selection of articles, the PICo strategy were used. Complete and original articles were included. To assist the analysis of the data, the information was operationalized in the IRAMUTEQ ${ }^{\circledR}$ software. 14 articles were selected, published in national and international journals, with a predominance of qualitative research. After a thorough analysis, two classes originated - Class 1: The Complexity paradigm and nursing care in the face of care adversities. Class 2: Complexity of care in the childhood and adolescence phases. Complexity Theory is used to understand the relationships and phenomena that surround nursing care as a whole.
\end{abstract}

Keywords: Nursing; Health; Nursing care; Comprehensive health care. 


\begin{abstract}
Resumen
El Objetivo de este estúdio fue identificar el uso del marco teórico de la Complejidad propuesto por Edgar Morin en la producción de conocimientos sobre el cuidado de enfermería. Se trata de una revisión integradora de la literatura, realizada en la Biblioteca Virtual en Salud y las bases de datos PubMed, de 2009 a 2020. Para la selección de artículos se utilizó la estrategia PICo. Se incluyeron artículos completos y originales. Para ayudar al análisis de los datos, la información se operacionalizó en el software IRAMUTEQ®. Se seleccionaron 14 artículos publicados en revistas nacionales e internacionales, con predominio de la investigación cualitativa. Luego de un análisis exhaustivo, se originaron dos clases - Clase 1: El paradigma de la Complejidad y el cuidado de enfermería ante las adversidades del cuidado. Clase 2: Complejidad del cuidado en las fases de niñez y adolescencia. La Teoría de la Complejidad se utiliza para comprender las relaciones y fenómenos que rodean al cuidado de enfermería en su conjunto.
\end{abstract}

Palabras clave: Enfermería; Salud; Atención de enfermería; Atención integral de salud.

\title{
1. Introdução
}

A Teoria da Complexidade, criada por Edgar Morin no início do século XX, introduz um novo conceito de pensamento, com base na teoria sistêmica, reforça a integralidade e a junção, com abominação às fragmentações e disjunções. Admite a complexidade do ser humano e a necessidade de ver o mundo em sua totalidade, interconectado, integrado com o modo de pensar e agir, opondo-se ao reducionismo (Morin, 2012; Costa et al., 2015).

A Complexidade traz, em sua base filosófica, o conceito da incerteza e da auto-organização como características marcantes do ser humano, que comporta a capacidade e aptidão para reunir, organizar, globalizar e reconhecer as singularidades de cada parte, com objetivo de distinguir e, simultaneamente, reunir as concepções do ser humano. Entretanto, os princípios da ordem e da lógica, não são excluídos, e sim integrados, articulando a ordem com a desordem, e a autonomia com a dependência (Morin, 2003, p. 69-78.).

A enfermagem, responsável pelo cuidado ao paciente, é permeada por experiências vivenciadas e interações, essas são apoiadas, dentro do contexto individual, coletivo e principalmente subjetivo. Durante seu processo de trabalho, o enfermeiro convive constantemente com experiências complexas, proveniente das reações comportamentais e vivenciadas pelo ser humano durante o processo de adoecimento. Nesta perspectiva, a Complexidade fomenta o aperfeiçoamento do cuidado, pautado no planejamento, nas relações assistenciais, no meio ambiente e nas interações com o paciente (Morin, 2008).

Todo o processo de cuidado, tem como foco as necessidades dos seres humanos, assim, a enfermagem auxilia na busca incessante pela aquisição do conhecimento e autocontrole, com intuito de manter o estabelecimento e preservação da vida, diante de todo a ressignificação e oscilações de conforto e desconforto. (Nascimento \& Erdmann, 2009; Hulley, Newman \& Cummings, 2015). Notoriamente, o cuidado é baseado na visão fragmentada do ser humano. Portanto, a Complexidade instiga a uma outra forma de entender a realidade, estimulando o pensamento que reúne, contextualiza, globaliza, mas que também seja capaz de compreender e reconhecer o singular, o individual e o concreto (Morin, 2008).

Considerando a complexidade do cuidado de enfermagem e a multidimensionalidade do ser humano é indispensável identificar como a literatura evidencia a relação com a Complexidade, pois, o cuidado de enfermagem não pode ser uma ação reducionista, mas um dinamismo de interações, reflexões e autoconhecimento. Este estudo tem como objetivo identificar a utilização do referencial teórico da Complexidade, proposto por Edgar Morin, na produção do conhecimento sobre cuidado de enfermagem.

\section{Metodologia}

\section{Tipo de Estudo}

Trata-se de uma revisão integrativa da literatura seguindo a abordagem proposta por Mendes, Silveira \& Galvão (2008). A questão de pesquisa foi elaborada de acordo com a estratégia População, fenômeno de Interesse e Contexto (PICo). Considerou-se a seguinte estrutura: P- enfermeiros; I - uso da teoria da Complexidade; Co - produção de conhecimento sobre 
cuidado de enfermagem. Dessa forma, elaborou-se a seguinte questão: Como os enfermeiros utilizam a teoria da Complexidade na produção de conhecimento sobre cuidado de enfermagem?

\section{Seleção dos Estudos}

Para os critérios de inclusão definiu-se: estudos originais, publicados nos últimos onze anos (novembro de 2009 a novembro de 2020), disponíveis eletronicamente na íntegra, sem restrições de idioma, localidade e método utilizado. Os critérios de exclusão foram: artigos que se distanciaram das reflexões propostas por este estudo, revisões de literatura/reflexão, editoriais, comunicação breve, projetos de ensaios clínicos, resumos de anais, teses, dissertações, trabalho de conclusão de curso, boletins epidemiológicos, relatórios de gestão, livros, documentos oficiais de programas.

\section{Coleta de Dados}

A coleta de dados ocorreu em dois momentos, primeiramente em setembro de 2019, posteriormente em novembro de 2020, nas fontes de informações: PubMed utilizando os termos contemplados no Medical Subject Headings (MeSH): Delivery of Health Care AND Nursing Care; e, Biblioteca Virtual em Saúde (BVS) com os Descritores em Ciências da Saúde (DeCS): Assistência à saúde AND cuidados de enfermagem e a junção das palavras chave teoria da complexidade/complexity theory e pensamento complexo. Estratégias de pesquisa abrangentes foram construídas para cada base de dados. Apresenta-se um exemplo no Quadro 1.

Quadro 1. Estratégia de busca aplicada em PubMed. Brasil, 2020.

((("delivery of health care"[MeSH Terms] OR ("delivery"[All Fields] AND "health"[All Fields] AND "care"[All Fields]) OR "delivery of health care"[All Fields]) AND ("nursing"[MeSH Subheading] OR "nursing"[All Fields] OR ("nursing"[All Fields] AND "care"[All Fields]) OR "nursing care"[All Fields] OR "nursing care"[MeSH Terms] OR ("nursing"[All Fields] AND "care"[All Fields])) AND (("complex"[All Fields] OR "complex s"[All Fields] OR "complexant"[All Fields] OR "complexants"[All Fields] OR "complexated"[All Fields] OR "complexation"[All Fields] OR "complexations"[All Fields] OR "complexe"[All Fields] OR "complexed"[All Fields] OR "complexes"[All Fields] OR "complexing"[All Fields] OR "complexities"[All Fields] OR "complexity"[All Fields] OR "complexs"[All Fields]) AND ("theorie"[All Fields] OR "theories"[All Fields] OR "theory"[All Fields] OR "theory s"[All Fields]))) OR ("nurse s"[All Fields] OR "nurses"[MeSH Terms] OR "nurses"[All Fields] OR "nurse"[All Fields] OR "nurses s"[All Fields]) NOT ("review"[Publication Type] OR "review literature as topic"[MeSH Terms] OR "review"[All Fields])

Fonte: Pubmed (2020).

Vale destacar no quadro acima (Quadro 1), a estratégia de busca completa de uma das bases de dados consultadas, incluindo os termos de busca, os operadores booleanos e/ou de proximidade, códigos de campo, filtros, parênteses e aspas para termos de busca com mais de uma palavra.

\section{Procedimentos de Análise e Tratamento dos Dados}

Para a análise dos artigos, foram excluídos os duplicados e aplicado os critérios de elegibilidade, na qual esses foram selecionados e identificados seguindo três etapas: 1) Leitura dos títulos e resumos dos estudos e exclusão daqueles que não se enquadraram nos critérios estabelecidos; 2) Leitura na íntegra dos artigos selecionados na primeira etapa; 3) Seleção das obras que se enquadraram nos critérios de elegibilidade. As etapas foram realizadas por duas pesquisadoras de forma independente, 
as discordâncias foram discutidas até que houvesse consenso. Os artigos duplicados nas bases foram excluídos.

Para a extração e síntese das informações contidas nos estudos selecionados, elaborou-se uma planilha no Excel com: identificação, título, ano de publicação, periódico, objetivo, design do estudo, principais resultados e conclusão.

As informações foram operacionalizadas pelo software IRAMUTEQ® (Souza et al., 2018). Para este estudo, adotouse a nuvem de palavras, a qual agrupa e organiza os vocábulos graficamente em função da sua frequência no texto, possibilitando a sua identificação a partir de um único arquivo, denominado corpus textual (Souza et al., 2018). A convergência entre os vocábulos destacados pelo software e a inferência dos dados, deram origem a duas classes definitivas.

\section{Aspectos Éticos}

Por tratar-se de revisão integrativa, o projeto não foi submetido ao Comitê de Ética em Pesquisa, no entanto, os dados e informações das publicações utilizadas no desenvolvimento deste estudo estão apresentadas de forma fidedigna.

\section{Resultados}

Constatou-se nas bases analisadas 2.430 obras, após a leitura minuciosa e a aplicação dos critérios de elegibilidade, restaram 14 artigos, os quais contemplam esta revisão integrativa (Figura 1).

Figura 1. Fluxograma da seleção dos artigos para esta revisão.

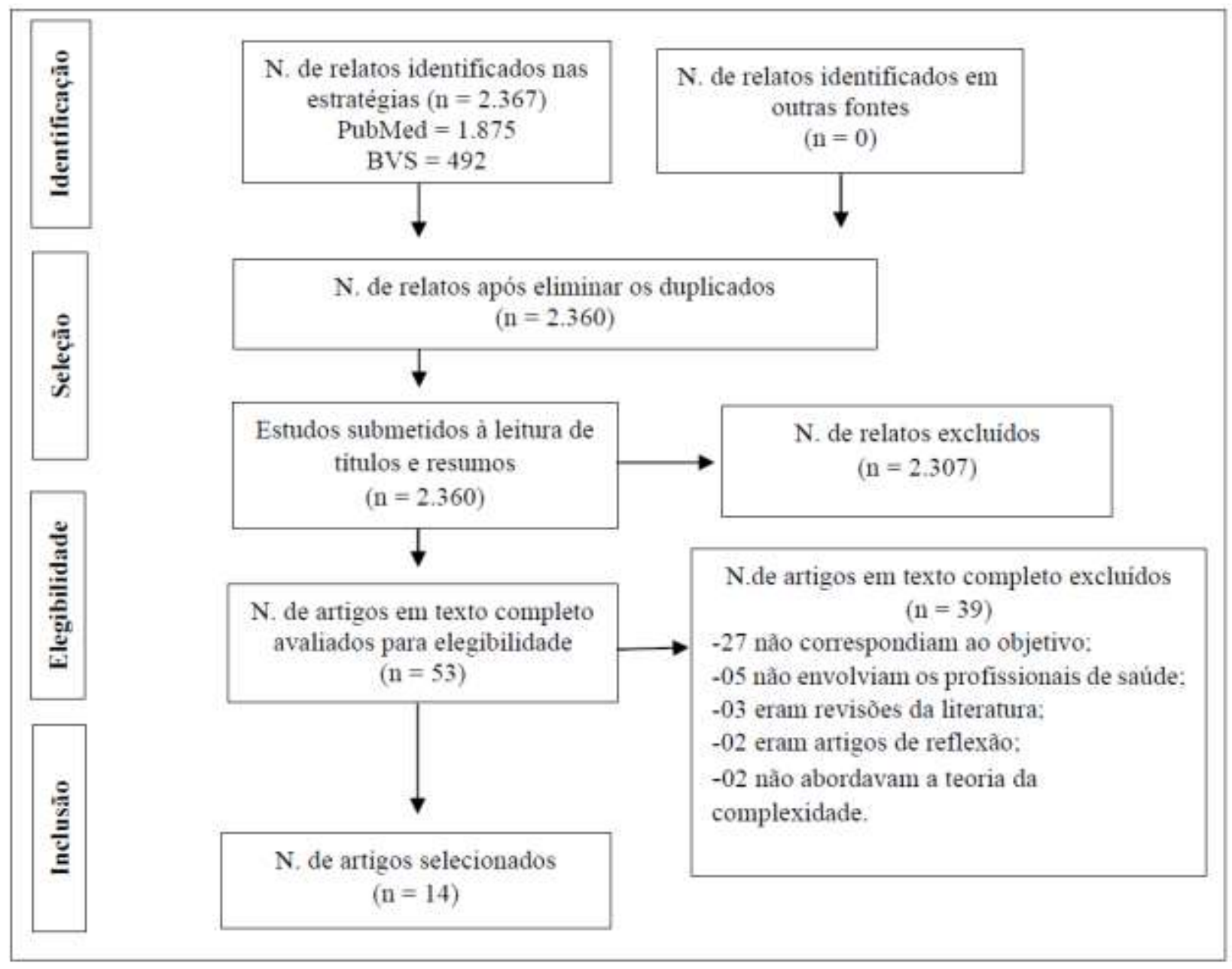

Fonte: Dados da revisão, PR (2020). 
A maioria dos estudos selecionados era de origem brasileira $(\mathrm{n}=09)$, os demais foram realizados na China, Itália, Reino Unido, Estados Unidos e Canadá. Todas as obras selecionadas eram de pesquisas qualitativas 100\% (n=14), e a maioria utilizou o referencial metodológico da Grounded Theory (Teoria Fundamentada nos dados) (n=13).

Conforme Quadro 2, destaca-se os principais resultados encontrados nos estudos selecionados para esta revisão.

Quadro 2. Caracterização dos artigos selecionados para esta revisão integrativa, no período de 2009 a 2020. Brasil.

\begin{tabular}{|c|c|}
\hline ID & Principais Achados \\
\hline $\begin{array}{l}\text { A01 } \\
\text { (Huang et al., } \\
\text { 2019) }\end{array}$ & $\begin{array}{l}\text { Apresenta três fatores relacionados a complexidade do cuidado, sendo aspectos do paciente como as } \\
\text { condições psicológicas, habilidade e idade; aspectos que envolvem a equipe de enfermagem, incluindo } \\
\text { experiências, educação permanente, competências e habilidades técnicas; e os fatores de organização e } \\
\text { equipamentos, que estão relacionados aos recursos humanos da equipe, processo de gestão e } \\
\text { multidisciplinaridade. }\end{array}$ \\
\hline $\begin{array}{l}\text { A02 } \\
\text { (Silva et al., } \\
\text { 2019) }\end{array}$ & $\begin{array}{l}\text { Relata as diversas percepções que os enfermeiros assistências tem em relação à pesquisa e o enfermeiro } \\
\text { pesquisador, em que muitas vezes é desvalorizado frente ao papel imprescindível que desempenha na } \\
\text { ciência. Concomitantemente o enfermeiro pesquisador reconhece as limitações dos enfermeiros } \\
\text { assistências frente a pesquisa e ciência. }\end{array}$ \\
\hline $\begin{array}{l}\text { A03 } \\
\text { (Prado et al., } \\
\text { 2019) }\end{array}$ & $\begin{array}{l}\text { Apresenta a comunicação como um importante desafio para o gerenciamento da enfermagem, } \\
\text { principalmente diante da terminalidade, momento único para a tríade paciente, família e profissionais de } \\
\text { saúde. A individualidade da situação requer do enfermeiro a capacidade de autogerenciamento do } \\
\text { cuidado, partindo dos princípios de ordem/desordem. }\end{array}$ \\
\hline $\begin{array}{l}\text { A04 } \\
\text { (Silva et al., } \\
\text { 2018) }\end{array}$ & $\begin{array}{l}\text { Revelam a necessidade dos profissionais de saúde em implementarem estratégias, que se adequem as } \\
\text { condições interação com as crianças e o familiar, visando principalmente técnicas lúdicas que amenizam } \\
\text { e facilitam o processo de avaliação e manejo da dor. }\end{array}$ \\
\hline $\begin{array}{l}\text { A05 } \\
\text { (Gordon et al., } \\
\text { 2018) }\end{array}$ & $\begin{array}{l}\text { O estudo apresenta a importância da liderança frente a um ambiente interprofissional complexo, na qual } \\
\text { há necessidade constante do formato de liderança ser negociado e renegociado, visto, a dinamicidade das } \\
\text { interações entre os profissionais. Dessa forma, é importante a redefinição dos conceitos de liderança por } \\
\text { profissionais de saúde educadores, a fim de proporcionar novas abordagens de pesquisa com foco na } \\
\text { liderança. }\end{array}$ \\
\hline $\begin{array}{l}\text { A06 } \\
\text { (Carlos et al., } \\
\text { 2017) }\end{array}$ & $\begin{array}{l}\text { Os resultados apontam a fragmentação dos cuidados em todas as etapas do processo assistencial que } \\
\text { envolvem as redes institucionais, o que enfatiza o desafio no processo de consolidação da assistência e a } \\
\text { complexidade do cuidado as famílias vítimas de violência contra a criança e adolescente. }\end{array}$ \\
\hline $\begin{array}{l}\text { A07 } \\
\text { (Ichikawa et } \\
\text { al., 2017) }\end{array}$ & $\begin{array}{l}\text { A teoria da Complexidade fornece subsídios para a compreensão do processo de luto as famílias, } \\
\text { principalmente a perda neonatal, pois, busca um olhar para o universo familiar como um todo, sem } \\
\text { fragmentação ou simplificando as relações, partindo do pressuposto de construção e a desconstrução da } \\
\text { família, na qual considera todas as influências recebidas, interna e externamente. }\end{array}$ \\
\hline $\begin{array}{l}\text { A08 } \\
\text { (Guarinoni et } \\
\text { al., 2015) }\end{array}$ & $\begin{array}{l}\text { A complexidade do cuidado assistencial do enfermeiro está imergidas em três categorias, sendo a } \\
\text { primeira voltada para o paciente, na qual envolve aspectos referentes a patologia, necessidades dos } \\
\text { cuidados (autonomia) e capacidade psicológica deste; outro aspecto é relacionado especificamente ao } \\
\text { enfermeiro, abrangendo o emocional, as habilidades técnicas e em alguns pontos a complexidade do } \\
\text { cuidado relaciona-se com a carga de trabalho; o último ponto enfatiza a organização, na qual engloba } \\
\text { fatores financeiros, burocráticos e a multidisciplinariedade. }\end{array}$ \\
\hline $\begin{array}{c}\text { A09 } \\
\text { (Carvalho, } \\
\text { Erdmann \& } \\
\text { Santana, 2015) }\end{array}$ & $\begin{array}{l}\text { Relata a adolescência como uma busca incessante pela autonomia, entretanto essa busca é enraizada pela } \\
\text { barreira que a dependência familiar proporciona, sendo necessário repentinas formulações da } \\
\text { autoimagem, da definição corporal e psicológica. Essa redefinição de autonomia é essencial para o } \\
\text { adolescente incorporar a capacidade de tomar próprias decisões e comparar a autonomia com a } \\
\text { maturidade, segurança e liberdade financeira. }\end{array}$ \\
\hline $\begin{array}{l}\text { A10 } \\
\text { (Silva et al., }\end{array}$ & $\begin{array}{l}\text { Os resultados apontam para a invulnerabilidade do adolescente em relação às práticas sexuais, assim, na } \\
\text { perspectiva do pensamento complexo, se dá a partir dos riscos/incertezas e ilusões que permeiam a } \\
\text { adolescência. O gerenciamento do cuidado de enfermagem ao adolescente está relacionado as }\end{array}$ \\
\hline
\end{tabular}




\begin{tabular}{|c|c|}
\hline 2015) & percepções que o enfermeiro tem do processo de adolescer. \\
\hline $\begin{array}{c}\text { A11 } \\
\text { (Pestana, } \\
\text { Erdmann \& } \\
\text { Souza, 2012) }\end{array}$ & $\begin{array}{l}\text { A categoria "Emergindo a complexidade do cuidado de enfermagem ao ser em ME" configura-se como } \\
\text { a consequência do modelo paradigmático intitulado "Desvelando relações e interações múltiplas do ser } \\
\text { enfermeiro na complexidade do cuidado ao ser em morte encefálica". É composta pelas subcategorias: } \\
\text { Emergindo os sentimentos e reações do ser enfermeiro no processo de cuidado ao ser em ME, } \\
\text { Apontando a complexidade do cuidado ao ser em ME e Avançando nas práticas de cuidado ao ser em } \\
\text { ME. O cuidado ao ser em morte encefálica é caracterizado por desordem e incertezas, fazendo com que } \\
\text { o enfermeiro vivencie sentimentos diversos e ambivalentes. A sua complexidade está em compreender a } \\
\text { sua singularidade e dialogicidade. }\end{array}$ \\
\hline $\begin{array}{c}\text { A12 } \\
\text { (Klock \& } \\
\text { Erdmann, } \\
\text { 2012) }\end{array}$ & $\begin{array}{l}\text { Ao considerar, a importância do conhecimento no cotidiano de UTIN, evidencia-se uma necessidade de } \\
\text { atualização profissional, motivada pelo surgimento de novas tecnologias que visam suprir as demandas } \\
\text { decorrentes dos avanços científicos e tecnológicos na assistência ao bebê prematuro. Visualizar o } \\
\text { cuidado do recém-nascido em UTIN em um modelo teórico explicativo implica em vislumbrar o cuidado } \\
\text { como sendo altamente complexo, sensível, singular e compartilhado, considerando a fragilidade do } \\
\text { Viver/Sobreviver do neonato em UTIN. }\end{array}$ \\
\hline $\begin{array}{l}\text { A13 } \\
\text { (Miller et al., } \\
\text { 2010) }\end{array}$ & $\begin{array}{l}\text { As práticas de atenção primária podem ser fecundamente entendidas como adaptativas complexas } \\
\text { sistemas que consistem em um núcleo (recursos-chave de uma prática, estrutura organizacional, e } \\
\text { processos funcionais), reserva adaptativa (recursos de prática que aumentam a resiliência identidade, } \\
\text { como relacionamentos) e atenção ao ambiente local. A eficácia desses atributos representa a capacidade } \\
\text { interna da prática. Com motivação adequada, práticas saudáveis e prósperas avançam ao longo de um } \\
\text { caminho de mudança de desenvolvimento lenta e contínua com períodos rápidos ocasionais de } \\
\text { transformação conforme eles evoluem melhor se adapta ao seu ambiente. Desenvolvimento de prática é } \\
\text { aprimorado através do uso sistemático de estratégias que envolvem a definição de direção e limites, } \\
\text { implementando sistemas de detecção, com foco em tensões criativas, e promovendo conversas de } \\
\text { aprendizagem. }\end{array}$ \\
\hline $\begin{array}{l}\text { A14 } \\
\quad(\text { Silva \& } \\
\text { Freitas, 2010) }\end{array}$ & $\begin{array}{l}\text { Compreender a complexidade hologramática do ser humano é vislumbrar que a parte está no todo e que } \\
\text { o todo está na parte. Os entrevistados relatam este cuidar, à medida que reconhecem e refletem sobre a } \\
\text { existência de um eu interior em si e nos clientes, verbalizando a complexidade hologramática do ser } \\
\text { humano. }\end{array}$ \\
\hline
\end{tabular}

Fonte: Autoras. Informações extraídas dos estudos desta revisão (2020).

No quadro acima, nota-se que os estudos discutem os cuidados de saúde/enfermagem atrelando-se as premissas do pensamento complexo e a complexidade envolta no cotidiano da assistência e das interações humanas.

A partir da organização dos dados no software IRAMUTEQ, pelo método da nuvem de palavras, os vocábulos Paciente, Cuidado e Enfermeiro obtiveram maior frequência no corpus, seguidos de Enfermagem, Saúde e Complexidade (Figura 2). 
Figura 2. Nuvem de palavras.

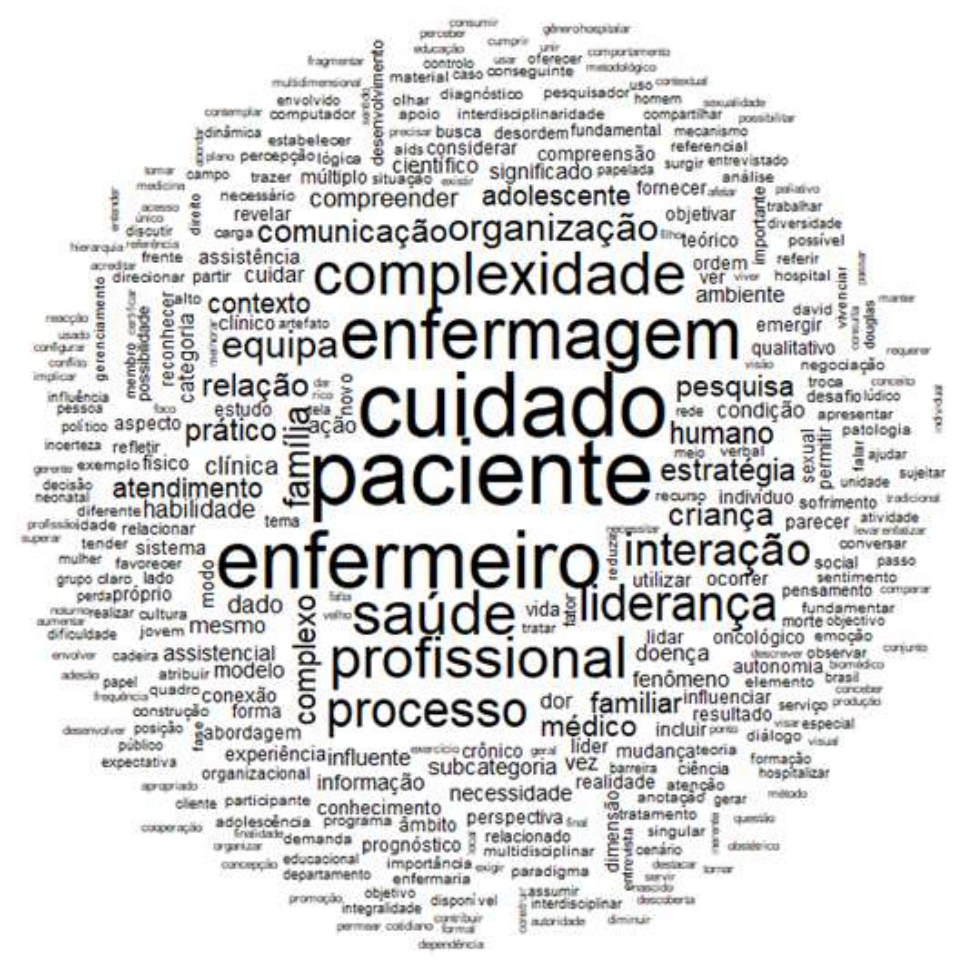

Fonte: Autoras. Vocábulos em destaque extraídos dos estudos desta pesquisa e organizados no software IRAMUTEQ® (2020).

O conteúdo foi dividido em cinco classes iniciais, cujos conteúdos foram minuciosamente analisados e organizados sistematicamente, dando origem a duas classes definitivas, denominadas: Classe 1: O paradigma da Complexidade e os cuidados de enfermagem diante as adversidades da assistência. Classe 2: Complexidade dos cuidados nas fases da infância e adolescência, apresentadas a seguir.

\section{Classe 1. O paradigma da Complexidade e os cuidados de enfermagem diante as adversidades da assistência}

Esta classe demonstra os principais aspectos dos cuidados de enfermagem frente as diversas situações, tais quais: a complexidade do ser humano; os desafios nos relacionamentos interpessoais e interprofissionais; a comunicação e as condutas da equipe multidisciplinar frente a terminalidade; a relação do ser humano/pessoa cuidada com a equipe de saúde, em particular, o enfermeiro; e, o desvelamento da complexidade do cuidado de enfermagem no âmbito da integralidade da assistência.

Nos estudos analisados, o ser humano, que está no papel de paciente, é descrito como um elemento complexo que possui necessidades objetivas que podem ser padronizadas e medidas (a patologia com suas características, as necessidades básicas afetadas, a dor), sendo compreendido como um sujeito único, que possui suas singularidades e particularidades relacionadas à sua cultura, emoções, desejos, significado que atribui à vida, à dor e à morte (Prado et al., 2019; Guarinoni et al., 2015; Pestana, Erdmann \& Souza, 2012; Silva \& Freitas, 2010).

Essa dualidade fica clara na definição de cuidado oferecido pelo enfermeiro intensivista: relacionando-se ao que é feito quando se trata de uma patologia e, ao mesmo tempo, mantém o cuidado centrado na pessoa. Além disso, a pessoa cuidada costuma ter o apoio familiar (ou a falta dele). Assim, a família é mencionada com muita frequência nas experiências dos enfermeiros como fator chave na determinação da complexidade do cuidado (Silva et al., 2019; Carlos et al., 2017; Guarinoni et al., 2015). 
Alguns artigos revelaram modos de enfrentamento adotados pelos profissionais de saúde para lidar com os desafios que emergem de seus relacionamentos interpessoais e interprofissionais nas relações de cuidado, especialmente, quando cuidam de crianças em estado grave. Desta forma, buscam a espiritualidade como estratégia para a resignação do cuidado (Silva et al., 2018; Carvalho, Erdmann \& Santana, 2015).

Outro aspecto abordado nos estudos foi a comunicação da equipe multidisciplinar sobre a terminalidade do ser humano e às condutas a serem ou não tomadas frente a casos terminais oncológicos e de morte encefálica (ME). Como fenômeno complexo, o processo de comunicação parece sofrer influência contextual, influenciando as interelações. Essa assertiva é valorosa para o gerenciamento do cuidado a qual permite compreender aspectos organizacionais que influenciavam a comunicação e, consequentemente, a qualidade da assistência à saúde (Pestana, Erdmann \& Souza, 2012).

A complexidade do cuidado em relação à ME encontra-se atrelada à mudança na concepção do Ser Uno (complexo) que exige os mesmos cuidados de um paciente grave, principalmente nos casos de potencial doador de órgãos. O desvelar do cuidado de enfermagem em ME implica promover uma mudança de paradigma na forma como este é visto e conduzido. Significa superar, indo muito além do paradigma reducionista que permite enxergá-lo como um morto, e incorporar um novo modo de cuidar, uma nova consciência e um olhar multidimensional, permitindo refletir e desconstruir as barreiras do cuidar tradicional e hegemônico (Miller et al., 2010).

No âmbito da complexidade, a assistência integral à saúde busca, ao mesmo tempo, separar e associar, comunicar os saberes científicos, e enraizar a esfera antropossocial na esfera biológica, por meio da contextualização, transcendendo os aspectos físicos da doença, que transpõe a ênfase do modelo biomédico/cartesiano (Ichikawa et al., 2017; Pestana, Erdmann \& Souza, 2012).

\section{Classe 2. Complexidade dos cuidados nas fases da infância e adolescência}

Nesta classe discute-se as estratégias de cuidados de enfermagem, sob a perspectiva da Complexidade para a promoção da saúde nas diferentes fases do desenvolvimento, principalmente, a infância e adolescência.

Estudo abarcou a complexidade do atendimento em saúde em relação à idade das pessoas cuidadas, o que influencia diretamente no humor e na comunicação com os membros da equipe de saúde. Jovens, geralmente se recuperam mais rapidamente, do que as pessoas de mais idade com doenças semelhantes. As vantagens dos jovens são suas habilidades de condicionamento físico, melhores mecanismos de defesa e comunicação, comparado com os idosos (Huang et al., 2019). Outros estudos abordaram aspectos sobre a saúde do adolescente, principalmente em relação à sexualidade, infecções sexualmente transmissíveis (IST) e a busca pela autonomia (Huang et al., 2019; Silva et al., 2019; Carvalho, Erdmann \& Santana, 2015; Silva et al., 2015; Miller et al., 2010).

Os enfermeiros reconheceram que a construção sociocultural dessas concepções se inicia antes mesmo do indivíduo adentrar a fase adulta da vida, uma vez que o adolescente, para cumprir os papéis de gênero, pode desempenhar práticas sexuais nocivas a si mesmo e ao outro. As conexões do processo de adolescer, numa perspectiva complexa, situam o adolescente entre ordens, desordens, interações e organizações necessárias ao seu desenvolvimento saudável. A complexidade envolvida nos fatores intervenientes ao exercício da sexualidade do adolescente direciona para a importância de estratégias pautadas na dinamicidade contextual, e singular de cada caso (Miller et al., 2010).

É pertinente a preocupação dos enfermeiros com os sentimentos de vulnerabilidade do adolescente em relação às práticas sexuais. Por conseguinte, faz se necessário compreender a relação hologramática da Complexidade para a reprodução de comportamentos que tangenciam o ser homem e o ser mulher na sociedade e no contexto da promoção da saúde (Silva et al., 2019; Carvalho, Erdmann \& Santana, 2015).

Um dos estudos destacou a importância da concepção de complexidade lógica - unitas multiplex (Carvalho, Erdmann 
\& Santana, 2015), referindo-se ao sistema de interações e a própria adolescência como um complexo fenômeno social, considerou a necessidade de investimentos que integram esse sistema e os demais que encontram-se diretamente relacionados, tais como a escola e a família, fomentando que a união desses sistemas, pode estabelecer mecanismos de ajuda ao adolescente com vistas à compreensão de que a saúde depende, também, de fatores intrínsecos ao sujeito (Carvalho, Erdmann \& Santana, 2015; Silva et al., 2015).

Em relação a autonomia, emerge durante essa ação/retroação no mundo das experiências vivenciadas com os adultos, pais e professores ou outros que assumam a responsabilidade desses jovens. Todo ser humano é aberto e age/retroage no ambiente em que vive. É na dimensão do ambiente social e afetivo que o adolescente passa uma fase de descobertas e de início da aquisição da independência. O ser autônomo é aquele que foge da ordem estabelecida para gerar uma diversidade de ações beneficiando a auto-organização do ambiente e reproduzindo o padrão organizacional com vistas a sua manutenção (Silva et al., 2015).

Ainda, um estudo abordou o lúdico como principal dispositivo de interação entre a equipe de saúde e a criança. Foi compreendido que o lúdico se configura como importante ferramenta de interação, cujos benefícios incluem: a redução do estresse, o fortalecimento da relação de confiança e a preparação da criança para o procedimento ao qual serão submetidas (Silva et al., 2018).

Desta forma, a partir do momento em que os pais compreendem a complexidade do diagnóstico e, consequentemente, vivenciam o final de vida de seu filho recém-nascido, inicia-se um processo delicado. Diante da perda neonatal, se faz necessário estabelecer relações de confiança com a equipe de saúde nas quais as informações sejam compartilhadas em tempo hábil e a comunicação clara, objetiva e compassiva viabilize que a família encontre espaço para contar e elaborar sua história. Ao entender o ser humano como um ser complexo, o cuidado direcionado a ele é também uma ação complexa, interdisciplinar, direcionando-se à transdisciplinaridade. Sendo o cuidado à família que sofre a perda do filho passível de ser abordado por meio da interdisciplinaridade e da transdisciplinaridade, como um cuidado complexo (Ichikawa et al., 2017).

\section{Discussão}

Os resultados deste estudo apontaram para a utilização da Teoria da Complexidade, na compreensão do papel do enfermeiro frente às diversidades complexas que permeiam o cuidado de enfermagem, bem como a demanda das relações que esta profissão gera ao profissional enfermeiro. Para, além disso, destaca-se a promoção de uma mudança de paradigmas na forma como o ser humano é visto e cuidado.

As pesquisas têm utilizado a Teoria da Complexidade, com intuito de compreender os fenômenos "complexus" dos seres humanos. Esse pensamento considera realidades e problemas polidisciplinares, transversais, multidimensionais, transnacionais, globais e planetários (Morin, 2008). Deste modo, com a análise dos estudos foi possível compreender e realçar os princípios e principais aspectos da Complexidade nos cuidados de enfermagem. Dessa forma, as diversas áreas utilizam a teoria, a fim de aclarar a compreensão de novas questões, cada vez mais complexas dentro dos fenômenos estudados.

No Brasil, as pesquisas foram realizadas no âmbito do Sistema Único de Saúde (SUS), em Unidades Básicas de Saúde e em Unidades Hospitalares. Estudos internacionais, utilizaram a Complexidade como lente, por meio da qual foi possível averiguar equívocos dentro de todo o processo de coordenação e execução da assistência de enfermagem. Dessa forma, esse referencial teórico visa estudar as relações humanas e seus comportamentos complexos, por isso foram utilizados nos estudos que abordavam a enfermagem em seu cotidiano de trabalho nas diversas instituições do sistema de saúde (Colon-Emeric et al., 2006; Piven et al., 2006). 
Assim, o termo que pode melhor definir o cuidado de enfermagem é a complexidade, a qual encontra-se inserida nos mais diversos contextos da assistência em saúde, sendo parte fundamental das organizações complexas que permeiam o cuidado e as interações com o paciente em suas diversas esferas ambíguas e paradoxais.

Ao reconhecer o cuidado de enfermagem como parte singular, individual e concreta da assistência à saúde, entende-se a necessidade de contemplar a construção sociocultural que tais ações apreciam, possibilitando a compreensão da realidade como fenômeno multidimensional o qual o enfermeiro faz parte, seja nas relações, nas interrelações e interconexões, formando uma integração de saberes e práticas (Morin, 2008). A busca pela produção do cuidado de forma qualificada pressupõe a necessidade de um olhar voltado ao Ser Uno (complexo) e multidimensional, compreendendo o indivíduo como um todo e em suas diferentes dimensões. Ademais, é preciso entender o cuidado de enfermagem como uma prática social e dinâmica, que reconhece as igualdades e diferenças biológicas, sociais, culturais e políticas, reconhecendo a complexidade da inter-relação do ser humano com as relações do processo de cuidado (Morin, 2001).

Tal fato corrobora com o conceito do Princípio Hologramático, que segundo Edgar Morin enfatiza "não apenas a parte está no todo, mas o todo está em cada uma das partes que compõe o todo" (Morin, 2008). Os resultados apontaram para a necessidade de romper com os mecanismos reducionistas, considerando a multidimensionalidade da prática de enfermagem e todas as influências recebidas, quer sejam intrínsecas ou extrínsecas. Assim, o princípio Hologramático, a forma individualista e fracionada de trabalho é rompida, permitindo que se compreenda as amplitudes das conexões e interconexões, a partir da interação entre indivíduos envoltos no processo, meio ambiente e práticas de saúde (Morin, 2008).

O Princípio Dialógico também foi amplamente trazido nos resultados desta investigação, quando se trata da articulação entre a importância de conviver com a diversidade de ideias e situações, compreendendo que a arte de cuidar do enfermeiro depende das relações entre ordens, desordens, interações e organização necessárias, mesmo que embora algumas vezes sejam antagônicas (Morin, 2007). Ao considerar a diversidade que o processo de cuidar em saúde se constitui, esse princípio parece permitir lidar com os conflitos, incertezas e instabilidades que a rotina da enfermagem está exposta.

Dessa forma, as práticas em saúde, principalmente o cuidado em enfermagem, podem ser respaldadas sob a ótica da Complexidade, quando considera-se a interdisciplinaridade nas ações, em que é almejado a promoção de olhares múltiplos para o fortalecimento e incentivo do cuidado integral, capaz de compreender a essência e realidade dos sistemas complexos dentro do cuidado em saúde, levando em consideração todas as penúrias impostas proveniente da diversidade terapêutica (Menossi \& Lima, 2004).

\section{Conclusão}

O uso da revisão integrativa como método para aprofundar os conhecimentos acerca da Teoria da Complexidade permitiu a compreensão do uso desse referencial nos cuidados de enfermagem. Isso porque, o cuidado de enfermagem é reconhecido como um fenômeno complexo e detém inúmeras interações entre indivíduos envolvidos no processo, meio ambiente e práticas em saúde.

Assim, percebeu-se neste estudo que a teoria da Complexidade é utilizada, principalmente, para compreender as relações e os fenômenos que circundam a assistência de enfermagem como um todo e em diferentes fases do desenvolvimento humano, sendo mais usual na atenção à criança e ao adolescente, pois esta é permeada de incertezas e inseguranças por parte dos profissionais, ressaltando a necessidade de a incorporação de múltiplos olhares para a execução do cuidado. Ainda, o estudo deu visibilidade aos conceitos do referencial utilizado pelos diferentes autores e suas possíveis aplicações no campo da assistência à saúde, isto é, ação-reflexão-ação.

Apreende-se que a prática de enfermagem está amplamente relacionada à complexidade do Ser e que o enfermeiro como um ser social que dispõe de conhecimentos técnicos científicos deve repensar novas formas de cuidado que abarquem a 
dinamicidade e a integralidade, fazendo articulações entre as redes de cuidado e as interconexões que dependem de si. Ainda, acredita-se que uma das principais contribuições dessa teoria seja a possibilidade de se constituir em um referencial norteador para apontar lacunas e possíveis estratégias para a reorganização das práticas em saúde.

Destaca-se como limitação deste estudo o tamanho reduzido da amostra, tendo em vista que, pesquisas relacionadas diretamente ao cuidado de enfermagem adotando o Pensamento Complexo, estão em constante crescimento. Ainda, a utilização e combinação das palavras chaves com os descritores podem ter contribuído para o resultado de apenas 14 artigos. Ademais, sugere-se trabalhos futuros que abarquem o trabalho multiprofissional e multidisciplinar em saúde, tanto nas instituições públicas e privadas como no domicílio. Ainda, que além do aprofundamento do tema para o levantamento de possíveis entraves no processo de cuidar, possa também trazer contribuições para estratégias de cuidado mais contextualizadas e dinâmicas na rotina dos serviços de saúde brasileiros.

\section{Agradecimentos}

O presente trabalho foi realizado com apoio da Coordenação de Aperfeiçoamento de Pessoal de Nível Superior Brasil (CAPES) - Código de Financiamento 001.

\section{Referências}

Carlos, D. M., Pádua, E. M. M., Silva, L. M. P., Silva, M. A. I., Marques, W. E. U., Leitão, M. N. D. C., \& Ferriani, M. D. G. C. (2017). The care network of the families involved in violence against children and adolescents: the Primary Health Care perspective. J Clin Nurs, $26(15-16)$, 2452-2467. https://doi.org/10.1111/jocn.13692

Carvalho, J. N., Erdmann, A. L., \& Santana, M. E. (2015). A dependência do outro na construção da autonomia do adolescente para o autocuidado. Cienc Cuid Saúde, 14(1), 910-916. https://doi.org/10.4025/cienccuidsaude.v14i1.16419

Colon-Emeric, C. S., Lekan-Rutledge, D., Utley-Smith, Q., Ammarell, N., Bailey, D., \& Piven, M. L. (2006). Connection, regulation, and care plan innovation: a case study of four nursing homes. Health Care Manage Rev, 31(4), 337-346. https://doi.org/10.1097 / 00004010-200610000-00009

Costa, M. C. M. D. R., Koerich, C., Ribeiro, J. C., Meirelles, B. H. S., \& Melo, A. L. S. F. (2015). Nursing care in view of complex thinking: integrative literature review. Revista Mineira de Enfermagem, 19(1), 180-7. http://www.dx.doi.org/10.5935/1415-2762.20150015

Gordon, L., Rees, C., Ker, J., \& Cleland, J. (2017). Using video-reflexive ethnography to capture the complexity of leadership enactment in the health care workplace. Adv in Health Sci Educ, 22, 1101-1121. https://doi.org/10.1007/s10459-016-9744-Z

Guarinoni, M., Petrucci, C., Lancia, L., \& Motta, P. C. (2015). The concept of care complexity: a qualitative study. J Public Health Res, 4(3), 588. https://doi.org/10.4081/jphr.2015.588.

Huang, B., Li, H., Chen, M., Lin, N., \& Wang, Z. (2019). Theoretical framework construction on care complexity in Chinese hospitals: A grounded theory study. International Journal of Nursing Sciences, 6, 192-197. https://doi.org/10.1016/j.ijnss.2019.03.011

Hulley, S. B., Newman, T. B., \& Cummings, S. R. (2015). Introdução: anatomia e fisiologia da pesquisa clínica. In: Hulley, S. B, (org.). Delineando a pesquisa clínica. Porto Alegre (RS): Artmed.

Ichikawa, C. R. F., Sampaio, P. S. S., Sá, N. N., Szylit, R., Santos, S. S. C., \& Vargas, D. (2017). O cuidado à família diante da perda neonatal: uma reflexão sob a ótica da teoria da complexidade. Rev enferm UFPE on line, 11(12), 5085-91. https://doi.org/10.5205/1981-8963-v11i12a22610p5085-5091-2017

Klock, P., \& Erdmann, A. L. (2012). Cuidando do recém-nascido em UTIN: convivendo com a fragilidade do viver/sobreviver à luz da complexidade. Rev Esc Enferm USP, 46(1), 45-51. http://dx.doi.org/10.1590/S0080-62342012000100006.

Mendes, K. D. S., Silveira, R. C. C. P., \& Galvão, C. M. (2008). Revisão integrativa: método de pesquisa para a incorporação de evidências na saúde e na enfermagem. Texto Contexto Enferm, 17(4), 758-64. https://doi.org/10.1590/S0104-07072008000400018

Menossi, M. J., \& Lima, R. A. G. (2004). A dor da criança e do adolescente com câncer: dimensões de seu cuidar. Rev Bras Enferm, 57(2), 178-82. https://doi.org/10.1590/S0034-71672004000200009

Miller, W. L., Crabtree, B. F., Nutting, P. A., Stange, K. C., \& Jaén, C. R. (2010). Primary Care Practice Development: A Relationship-Centered Approach. Ann Fam Med, 8, Suppl 1, s68-s79. https://doi.org/10.1370/afm.1089

Morin, E. (2001). O Método 2: a vida da vida. Sulina.

Morin, E. (2003). Representação e complexidade. In: Mendes C, Editor. A necessidade de um pensamento complexo. Rio de Janeiro: Garamond.

Morin, E. (2007). O método 6: ética. (3a ed), Sulina. 
Research, Society and Development, v. 10, n. 2, e5810212253, 2021

(CC BY 4.0) | ISSN 2525-3409 | DOI: http://dx.doi.org/10.33448/rsd-v10i2.12253

Morin, E. (2008). Introdução ao pensamento complexo. (5a ed), Instituto Piaget.

Morin, E. (2012). A cabeça bem-feita: repensar a reforma, reformar o pensamento. (2a ed.), Bertrand Brasil.

Nascimento, K. C., \& Erdmann, A. L. (2009). Understanding the dimensions of intensive care: transpersonal caring and complexity theories. Rev Latino-Am Enferm, 17(2), 215-21. http://dx.doi.org/10.1590/S0104-11692009000200012.

Pestana, A. L., Erdmann, A. L., \& Sousa, F. G. M. (2012). Emergindo a complexidade do cuidado de enfermagem ao ser em morte encefálica. Esc Anna Nery (impr.), 16(4), 734-740. http://dx.doi.org/10.1590/S1414-81452012000400013

Piven, M. L., Ammarell, N., Bailey, D., Corazzini, K., Colon-Emeric, C. S., \& Lekan- Rutledge, D. (2006). MSD Coordinator relationships and nursing home care processes. West J Nurs Res, 28(3), 294-309. https://doi.org/10.1177/0193945905284710

Prado, R. T., Leite, J. L., Silva, Í. R., \& Silva, L. J. (2019). Comunicação no gerenciamento do cuidado de enfermagem diante do processo de morte e morrer. Texto Contexto Enferm, 28, e20170336. http://dx.doi.org/10.1590/1980-265X-TCE-2017-0336

Silva, A. L., \& Freitas, M. G. (2010). O ensino do cuidar na Graduação em Enfermagem sob a perspectiva da complexidade. Rev Esc Enferm USP, 44(3), 68793. http://dx.doi.org/10.1590/S0080-62342010000300019

Silva, Í. R., Gomes, A. M. T., Valadares, G. V., Santos, N. L. P., Silva, T. P., \& Leite, J. L. (2015). Percepções de enfermeiros acerca das vulnerabilidades para DST/Aids diante das conexões do processo de adolescer. Rev Gaúcha Enferm, 36(3), 72-78. http://dx.doi.org/10.1590/1983-1447.2015.03.47293

Silva, I. R., Silva, T. P., Lins, S. M. S. B., Silva, L. J., \& Leite, J. L. (2019). Nurse researchers and nursing assistants: construction and projection of polymorphous identities. Rev Bras Enferm, 72, Suppl 1, 204-12. http://dx.doi.org/10.1590/0034-7167-2017-0871

Silva, T. P., Leite, J. L., Stinson, J., Lalloo, C., Silva, Í. R., \& Jibb, L. (2018). Action and interaction strategies for the care of hospitalized children with chronic cancer pain. Texto Contexto Enferm, 27(4), e3990017. http://dx.doi.org/10.1590/0104-07072018003990017

Souza, M. A. R., Wall, M. L., Thuler, A. C. M. C., Lowen, I. M. V., \& Peres, A. M. (2018). The use of IRAMUTEQ software for data analysis in qualitative research. Rev Esc Enferm USP, 52, e03353. http://dx.doi.org/10.1590/S1980-220X2017015003353 